\title{
Choosing a Model for eConsult Specialist Remuneration: Factors to Consider
}

\author{
Clare Liddy ${ }^{1,2, *}$, Catherine Deri Armstrong ${ }^{3}$, Fanny McKellips ${ }^{1}$, Paul Drosinis ${ }^{1}$, \\ Amir Afkham ${ }^{4}$ and Erin Keely ${ }^{5}$ \\ 1 C.T. Lamont Primary Health Care Research Centre, Bruyère Research Institute, Ottawa, ON K1R 6M1, \\ Canada; fmckellips@bruyere.org (F.M.); pdrosinis@bruyere.org (P.D.) \\ 2 Department of Family Medicine, University of Ottawa, Ottawa, ON K1R 6M1, Canada \\ 3 Department of Economics, University of Ottawa, Ottawa, Ontario K1N 6N5, Canada; \\ CDArmstrong@uottawa.ca \\ 4 Champlain Local Health Integration Network, Ottawa, ON K1J 1A3, Canada; Amir.Afkham@lhins.on.ca \\ 5 Division of Endocrinology and Metabolism, The Ottawa Hospital, Ottawa, ON K1H 7W9, Canada; \\ ekeely@toh.on.ca \\ * Correspondence: cliddy@bruyere.org; Tel.: +1-613-562-6262 (ext. 2928) \\ Academic Editor: Francisco Falcone \\ Received: 8 April 2016; Accepted: 13 June 2016; Published: 18 June 2016
}

\begin{abstract}
Electronic consultation (eConsult) is an innovative solution that allows specialists and primary care providers to communicate electronically, improving access to specialist care. Understanding the cost implications of different remuneration models available to pay specialists is of critical importance as adoption of these services continues to increase. We used data collected through the Champlain BASE (Building Access to Specialists through eConsultation) eConsult service to simulate the cost implications of different remuneration models in Canada. The prorated hourly rate model averaged \$45.72 CAD (Canadian Dollar) per eConsult while the prorated hourly rate with incentive averaged $\$ 51.90$ CAD per eConsult, and the fee for service cost $\$ 60.50$ CAD per eConsult. Paying all specialty groups to block three hours per week for eConsults averaged \$337.44 CAD per eConsult and paying for 1-h blocks averaged \$133.41 CAD per eConsult. As the remuneration of specialists is the largest cost driver of an established eConsult service, our findings can inform policymakers considering the implementation of eConsult or wishing to further develop an existing service.
\end{abstract}

Keywords: access to care; health services research; primary care; wait times

\section{Introduction}

Prompt access to specialist care is a major challenge facing the health care system. According to a report from the Fraser Institute, wait times for specialist care in Canada grew 132\% between 1997 and 2013 [1]. Issues of poor access to specialist care are not limited to Canada; many healthcare systems around the world face similar problems [2]. In an effort to increase access to specialist care and decrease wait times, several countries have implemented electronic consultation (eConsult) services [3-5]. These services enable primary care providers (PCPs) and specialists to communicate electronically over a secure web platform in order to discuss a specific patient's care. Even when the specialist must see the patient, a previous eConsult can increase the visit's effectiveness [6]. eConsult services have been shown to decrease wait times [7], improve care coordination and collegiality between primary and specialty care [8], avoid unnecessary face-to-face specialist visits [9], and provide educational opportunities for both PCPs and specialists [10]. Importantly, eConsult systems have shown great potential to reduce costs and lead to greater overall health system efficiency $[5,11]$. 
Specialist remuneration is the main cost driver in any (established) eConsult initiative and thus plays a crucial role in any cost or efficiency calculation. While eConsult is being implemented in a variety of regions across the world including the United States [12], Finland [5], and Canada [13], there is considerable variation in the types of remunerations schemes being used. Examples of eConsult specialist remuneration schemes include fee-for-service (Alberta), the use of salaried physician specialists (San Francisco General Hospital) [14], work load credit (Veterans Affairs system and Mayo Clinic) [15,16], and pro-rated hourly (Champlain) [17]. It is understood that the method of remuneration can have important implications on service quality, provider engagement and importantly, cost. The implementation and sustainability of any such service relies crucially on cost considerations.

Since 2010, we have been operating the Champlain BASE (Building Access to Specialists through eConsultation) eConsult service in our health region of Eastern Ontario, Canada. Over 14,000 eConsults have been submitted to many of the 86 different specialty groups available to users of the service. We have routinely collected system utilization data since the launch and have a unique opportunity to explore and compare the impact different remuneration models can have on the service.

The purpose of this study is to understand the cost implications of different remuneration models. We use data collected through the service to compare the costs that would be paid to specialists under four separate remuneration models. Our findings will be of interest to other regions interested in implementing eConsult services in order to inform decision making on the most appropriate compensation model.

\section{Materials and Methods}

\subsection{Study Design}

This is simulation study of all cases submitted over a one-year period by PCPs registered to use the Champlain BASE eConsult service.

\subsection{Setting}

The Champlain Region is one of the province's fourteen regional health districts and includes the city of Ottawa and its surrounding rural communities. Approximately half of the 1.2 million people who live in the region reside in Ottawa, which acts as a primary referral center for outlying rural communities [18].

In Canada, the health care system is publicly funded and available freely to all Canadians. A variety of payment models for physicians are used including traditional fee-for-service, capitation, and blended models. In Ontario, the majority of specialists are remunerated using a fee-for-service model, whereas PCPs operate in capitation or blended models. Access to a specialist requires a referral from a PCP or other physician, although only the specialist is remunerated for the referral.

\subsection{The Champlain BASE eConsult Service}

The Champlain BASE eConsult service is a secure, web-based platform allowing online communication between PCPs and specialists, reducing the need for face-to-face referrals to specialists [6,13]. PCPs who wish to consult a specialist fill out an electronic form with patient history and their detailed clinical question, with the option of attaching supplementary files or images if deemed necessary. The case is then assigned to a specialist, who is expected to respond within one week. Specialists are remunerated quarterly at $\$ 200$ per hour prorated to the self-reported time ( $<10 \mathrm{~min}, 10-15 \mathrm{~min}, 15-20 \mathrm{~min}$, and $>20 \mathrm{~min}$ ) they spent answering eConsults [17]. Similar to the traditional referral there is no billing for initiating an eConsult, but the specialists receiving and subsequently answering the eConsult are paid. The chosen $\$ 200 /$ hour rate was based on average remuneration of specialists in the province of Ontario [17]. Specialist remuneration ranges depending on the specialty type, anywhere from $\$ 50.00$ per consult for Radiology to $\$ 199.40$ per consult for 
Psychiatry [19]. According to a survey of specialists registered to use the Champlain BASE eConsult service, most specialist ( $88 \%$ ) are satisfied with the uniform payment model, despite variability of payment fees amongst specialty groups [20].

\subsection{Data Collection}

The data used in this study is routinely collected through the eConsult service and includes the total number of eConsults submitted and to which specialty, the date and time stamps of the PCP and specialist responses, and self-reported time for specialists to complete the eConsult. All eConsults completed between 1 April 2014 and 31 March 2015 were included in this analysis, representing the fourth year of our service.

For the purposes of this simulation study, our analysis included only eConsults that were submitted to medical specialties that PCPs would refer to in a traditional setting. We excluded all eConsults directed to the following specialty groups: bariatric care, cancer screening, chiropody, clinical pharmacist, diabetes education, HIV pharmacist, and radiology.

\subsection{The Remuneration Models}

We chose to examine four separate remuneration models that are either currently in existence or are being tested in our province as potential ways to pay for eConsults:

1. Prorated hourly rate: Specialists are compensated $\$ 200$ per hour prorated to the length of time it takes them to complete each eConsult. This model is currently used to remunerate specialists who use the Champlain BASE eConsult service and has been in existence since project inception in 2009.

2. Incentive model: On top of the standard prorated hourly rate as above, specialists receive a $\$ 10$ bonus for eConsults completed within $24 \mathrm{~h}$ and a $\$ 5$ bonus for eConsults completed within $48 \mathrm{~h}$. An incentive model is currently being tested for similar eConsult services operating within our province of Ontario.

3. Fee-for-Service (Billing code): Specialists are paid a flat fee of $\$ 44.50$ per eConsult, regardless of how long it takes to complete. This fee code was based on an existing store-forward telemedicine fee code. Additionally, PCPs are remunerated $\$ 16.00$ for the referral. These fees are based on existing fee codes in Ontario [19].

4. Flat weekly fee: Specialists block off a set time each week to devote to answering eConsults. The costs were calculated under two scenarios: (1) each specialty blocks one three-hour period per week at $\$ 200$ per hour regardless of the number of eConsults they are assigned; or (2) each specialty blocks a minimum of one hour, with the option to increase in one hour increments, if the volume of assigned eConsults is high enough.

\subsection{Analysis}

We calculated the total and per-eConsult costs separately under each remuneration model for all cases completed during the study period. For the prorated hourly rate model, the time specialists reported taking to answer cases was summed and assessed at a prorated hourly rate of $\$ 200$. In the incentive model, we used the cost of the prorated hourly rate model and added the cost for bonuses for all eConsults completed within one and two days ( $\$ 10$ and $\$ 5$ for each, respectively). For the physician billing code model, we simply assigned the cost of $\$ 60.50$ for every eConsult that occurred during the study period to remunerate both PCPs (\$16.00) and specialists (\$44.50). While a fee code for PCPs exists in Ontario, this is the only model in our study that remunerates PCPs, increasing the cost of this model. As a sensitivity analysis, we have calculated the costs of this model while excluding PCP remuneration to facilitate comparison with other models.

For the flat weekly sessional fee model, we tallied the number of active specialty groups and calculated costs under two scenarios. First, we assumed that all specialties would block off three hours 
each week in order to mimic booked clinical time and calculated the cost of paying each $\$ 600$ per week for 48 weeks (in order to allow for vacation time). However, when the volume of cases per week is low, this model would not be feasible from a cost perspective. We therefore modified the model and assumed all specialties would be paid a base salary of one-hour per week, with the option to increase if the volume was high enough, but only by one-hour increments. To do this, we calculated the average number of eConsults completed per specialty per week over the study period. We assumed each case takes fifteen minutes to complete (i.e., four eConsults per hour), which is the median of the time specialists spent to complete eConsults during our study period. If the average weekly number of eConsults was less than four the specialty group received $\$ 200 /$ week, if the average was between four and eight the specialty group received $\$ 400 /$ week, and specialty groups with over eight eConsults per week on average received $\$ 600 /$ week.

In the flat weekly fee model, if specialists devote a set block of time each week to answer eConsults, this may negatively impact response times. Given that a key benefit of eConsult is its expedited response time, we estimated how much of an impact there might be under this assumption. To do so, we used the most popular day of the week for specialists to respond to eConsults and assumed that all specialists would respond on that day only (i.e., if the eConsult is submitted on a Monday and the set time is also on Monday, we assumed the specialist would respond on the same day). We calculated response times under this assumption and compared it to the actual response times observed in the pro-rated model to understand how large the increase would be.

To provide comparability between the flat weekly sessional fee model and the prorated hourly rate model, we determined the volume of eConsults required for both remuneration models to have equal costs. This was done by dividing the total cost of the flat weekly sessional fee model by the average cost per eConsult of the prorated hourly rate model. Dividing again by the number of specialties gives the average volume of eConsults each specialty would have to receive to for both remuneration models to have the same cost.

\section{Results}

A total of 3775 eConsults were completed between 1 April 2014 and 31 March 2015. We excluded 105 eConsults submitted to specialties that PCPs would not typically refer to in a traditional setting, leaving a total of 3670 (97.2\% of the total) eConsults submitted to 68 specialists from 43 different specialty groups (Figure 1).

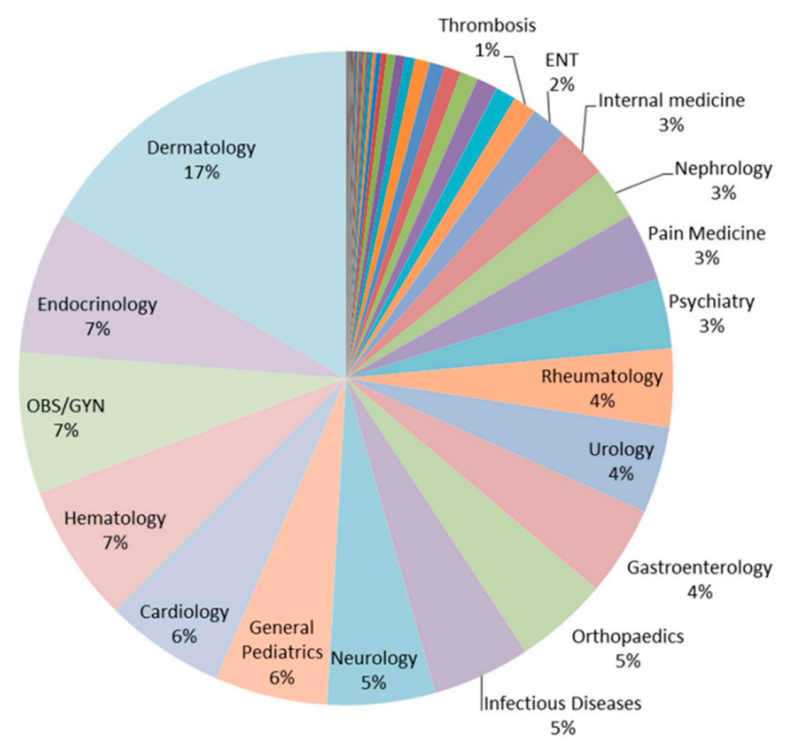

Figure 1. Specialty distribution for all cases completed between 1 April 2014 and 31 March 2015 $(n=3670)$. ENT = ears, nose, and throat; OBS/GYN = Obstetrics and gynecology. 
Specialists answered a median of 37 (Interquartile range 6-63) eConsults during the study period. PCPs submitted eConsults most frequently on Tuesdays (21.4\%) while specialists tended to reply most often on Wednesdays (19.5\%) (Table 1). The median specialist response time from receipt of request was 0.78 days (IQR: $0.15-3.2$ ) while the average was 2.2 days (standard deviation $=3.1$ ). The self-reported time it took the specialist to complete an eConsult was under ten minutes in $49 \%$ of cases, ten to fifteen minutes in $32 \%$ of cases, fifteen to twenty minutes in $15 \%$ of cases, and over twenty minutes in $4 \%$ of cases.

Table 1. Utilization characteristics of eConsults submitted between 1 April 2014 and 31 March 2015.

\begin{tabular}{cc}
\hline \multicolumn{2}{c}{ Distribution $(\boldsymbol{n}=\mathbf{3 6 7 0})$} \\
\hline Median Specialist Response Time (in Days) & $\mathbf{0 . 7 8}$ (IQR: 0.15-3.2) \\
\hline Time to Complete eConsult & Percentage $(\boldsymbol{n})$ \\
$<10$ mins & $48.8(1790)$ \\
$10-15$ mins & $32.0(1175)$ \\
$15-20$ mins & $15.3(563)$ \\
$>20$ mins & $3.9(142)$ \\
\hline Day of Week Submitted & Percentage $(\boldsymbol{n})$ \\
\hline Sun & $4.3(156)$ \\
Mon & $17.9(656)$ \\
Tue & $21.4(785)$ \\
Wed & $19.4(711)$ \\
Thu & $20.4(749)$ \\
Fri & $13.8(505)$ \\
Sat & $2.9(108)$ \\
\hline Day of Week Answered * & Percentage $(n)$ \\
Sun & $7.5(277)$ \\
Mon & $18.2(669)$ \\
Tue & $17.3(636)$ \\
Wed & $19.7(724)$ \\
Thu & $17.5(643)$ \\
Fri & $13.1(481)$ \\
Sat & $6.5(239)$ \\
\hline
\end{tabular}

* Time stamp unavailable in one instance of specialist response.

Table 2 shows the total and average estimated costs for specialist remuneration under each model. The total cost of remunerating specialists using the prorated hourly rate model was $\$ 167,783$. Under this model, the average cost per eConsult was $\$ 45.72$. The incentive payments calculated for the incentive model had a value of $\$ 22,705$, which brings the total cost of the incentive model to $\$ 190,488$ and increases the average cost per eConsult by $13.5 \%$ to $\$ 51.90$. Of the 68 specialists included in the study, 63 would have received an incentive payment at least once. Of the 3,670 eConsults examined, $67.5 \%$ would have been eligible for an incentive payment, since $56.2 \%(n=2062)$ were completed within one day and $11.4 \%(n=417)$ within two days. Table 3 shows a specialty breakdown of the distribution of eConsults that were completed within one and two days, for the ten most popular specialties. The cost of the fee-for-service model came to $\$ 222,035$. In this model, the cost per eConsult is $\$ 60.50$. When excluding the PCP remuneration, the cost of the fee-for-service model was $\$ 148,635$. Using the flat weekly sessional fee to calculate remuneration costs brought the total to $\$ 1,238,400$. Under this model, the average cost per eConsult is $\$ 337$. However, if we assume that all specialty groups would receive at least $\$ 200$ per week and then rounding up the costs to the next hour, the total cost becomes $\$ 489,600$, or $\$ 133.41$ per eConsult. Under this modified flat weekly fee model, only one specialty group (dermatology) received enough eConsults to warrant a three hour time block per week. The average cost per eConsults for dermatology cases $(n=611)$ under this model was $\$ 47.14$, which is lower than the average cost per eConsults for dermatology under the prorated hourly rate model, 
which was $\$ 48.15$. Among the remaining specialty groups, $14 \%(n=6)$ would receive $\$ 400$ per week and $84 \%(n=36)$ would receive $\$ 200$ per week. Assuming that all specialists would only respond to eConsults on Wednesdays, the median response time from assignment increases substantially from 0.78 to 5.13 days. Each specialty would have to receive approximately 630 eConsults a year for the prorated hourly rate and flat weekly sessional fee models to have the same cost.

Table 2. Total cost and average cost per eConsult for different remuneration models.

\begin{tabular}{ccc}
\hline Model & Cost (\$) & Average Cost per eConsult (\$) \\
\hline Prorated hourly rate & 167,783 & 45.72 \\
Prorated hourly rate + incentive & 190,488 & 51.90 \\
Fee-for-service & 222,035 & 60.50 \\
Flat weekly sessional fee & - & - \\
Booked clinic time & $1,238,400$ & 337.44 \\
1-h increments & 489,600 & 133.41 \\
\hline
\end{tabular}

Table 3. Distribution of the time to complete eConsult for the ten most popular specialties.

\begin{tabular}{cccc}
\hline Specialty & $<\mathbf{1}$ Day & $<2$ Days & Total \\
\hline Dermatology & $90.67 \%(554)$ & $6.55 \%(40)$ & 611 \\
Endocrinology & $78.33 \%(206)$ & $12.17 \%(32)$ & 263 \\
OBS/GYN & $37.50 \%(96)$ & $16.41 \%(42)$ & 256 \\
Hematology & $29.08 \%(73)$ & $11.55 \%(29)$ & 251 \\
Cardiology & $40.18 \%(88)$ & $14.16 \%(31)$ & 219 \\
General Pediatrics & $42.72 \%(88)$ & $9.71 \%(20)$ & 206 \\
Neurology & $78.06 \%(153)$ & $6.63 \%(13)$ & 196 \\
Infectious Diseases & $62.56 \%(122)$ & $7.18 \%(14)$ & 195 \\
Orthopaedics & $24.86 \%(43)$ & $13.87 \%(24)$ & 173 \\
Gastroenterology & $38.04 \%(62)$ & $14.72 \%(24)$ & 163 \\
\hline
\end{tabular}

\section{Discussion}

The results of our study demonstrate that choosing a compensation model for remuneration within an eConsult service can have large cost implications. This study is novel in that it used data collected through a fully established, multi-specialty eConsult service. To our knowledge this is the first study to compare different remuneration models, an important consideration for any health region interested in implementing a similar service.

Our findings suggest that the prorated hourly rate model is the most cost effective in terms of average cost per eConsult (\$45.72). Although it raises the expenditure, the incentive model may be considered if promptness of response is a priority (Table 4). It is important to note that the current analysis does not adjust for the likely possibility that bonuses would have induced specialists to respond even faster than they did. However, specialists in the Champlain BASE model were not subject to an incentive and yet over two thirds of eConsults were completed in less than two days, suggesting that specialists do not need an incentive to respond promptly. Given that eConsults in our region are not intended to be used in urgent cases, the extra payment for quick responses seem unnecessary; however, other regions where rapid response is required could use a payment incentive to achieve this goal. When excluding remuneration for the $\mathrm{PCP}$, the fee-for-service model led to a slightly lower cost than the prorated hourly rate model. This is because the fee is slightly lower than the average cost of an eConsult under the prorated hourly rate model. This model does not account for the complexity of cases and the cost savings result from a lower payment to the specialists.

The flat weekly sessional fee model was effective from a cost perspective for dermatology, which is the specialty with the highest demand. One important caveat is that this estimate assumes that one specialist would respond to all cases and does not include the number of specialists registered to actually respond to eConsult. In reality, more than one dermatologist would be needed to respond to 
this volume of cases. This would make the costs for the model much higher if the flat fee was paid to each individual. Furthermore, where the demand is much lower among certain specialty groups, the flat weekly fees translate to substantially higher costs per case, as certain specialties would be on stand-by and would still get paid even if no eConsults are submitted. These findings suggest that flat weekly remuneration strategies do not make sense given the large heterogeneity in the demand for, and length of time taken to respond to, eConsults. While the advantage of this model is that it gives the specialist a protected time period to devote to eConsult, the consequence is that it delays the response time, limits flexibility, and does not recognize individual case complexity that may result in a back and forth between the PCP and specialist.

Table 4. Pros and cons to consider when deciding on which remuneration model to implement for eConsult.

\begin{tabular}{cll}
\hline Model & \multicolumn{1}{c}{ Pros } & \multicolumn{1}{c}{ Cons } \\
\hline Prorated hourly rate & $\begin{array}{l}\text { Allows for more specialty services to be } \\
\text { available and payment only when specialist } \\
\text { responds; Allows for flexibility with respect to } \\
\text { case volume and complexity }\end{array}$ & $\begin{array}{l}\text { Managed service that is separate from usual } \\
\text { funding model }\end{array}$ \\
\hline $\begin{array}{c}\text { Prorated hourly rate } \\
\text { with incentive }\end{array}$ & $\begin{array}{l}\text { Has the potential to promote faster responses } \\
\text { from specialists }\end{array}$ & $\begin{array}{l}\text { Quality of the specialist response may be lower; } \\
\text { Questionable added cost given: non-urgent } \\
\text { nature of eConsult; measured median [average } \\
\text { response intervals without the incentive }\end{array}$ \\
\hline Physician billing code & $\begin{array}{l}\text { More aligned with usual payment for } \\
\text { traditional consultations }\end{array}$ & $\begin{array}{l}\text { Does not take into account case complexity; } \\
\text { Physicians have to complete the billing process } \\
\text { themselves; Less flexibility when addressing } \\
\text { payment for non-physician specialties }\end{array}$ \\
\hline Flat weekly sessional fee & $\begin{array}{l}\text { Simplified planning and payment mechanism } \\
\text { for specialists and payer }\end{array}$ & $\begin{array}{l}\text { Not cost-efficient for low-volume specialties; } \\
\text { May negatively impact response time }\end{array}$ \\
\hline
\end{tabular}

One potential solution to consider as the number of eConsults submitted increases, is to implement a hybrid model in which different payment models are used for different specialty groups depending on volume. The most commonly used specialty groups would be remunerated via the flat weekly fee model, while the less commonly used could be paid either via a prorated or fee-for-service amount. Alternatively, if a standardized remuneration model is desired, one solution that has been proposed to reduce costs using a flat fee per week model would be to eliminate the less frequently used specialty groups and limit the service to a smaller selection of the most common specialties. However, this would have two important implications for PCPs and patients. First, the ability to have a rich suite of specialties increase's the PCPs' confidence that a specialty applicable to their case would be available. With more specialty groups available, PCPs may be more likely to think of eConsult and less likely to doubt its applicability in a given case. Second, patients requiring care from the less frequently used specialties would not benefit from timely care.

In some fee-for service models, PCPs are remunerated for creating an eConsult. To our knowledge, only one study has examined the time investment required to submit a teledermatology eConsult and the authors reported that submitting an eConsult increased the average time of a GP visit, and this was due primarily to having to fill out the electronic referral form [21]. There are ways to work around this: within our service, we have created a delegate user feature that allows the PCP to identify a delegate (typically medical office staff or a referral clerk) who is set up to submit the eConsult request on behalf of the PCP, thereby reducing the PCP's work load. Similarly, the electronic form within the San Francisco General Hospital's eReferral service automatically populates with relevant PCP and patient information, which shortens the length of time required to complete the form [7]. Future research should focus on the impact of eConsult on workflow and this should be done within the lens of overall office efficiency and not only from a provider perspective. Health service organizations who widely adopt eConsult should see greater overall efficiency due to a reduction in the number of traditional face-to-face referrals that are processed. 
Remuneration models likely play a role in the adoption of eConsult by specialists and potentially PCPs. A recent survey found that specialists were largely satisfied with the amount and model of remuneration, and that a uniform price was acceptable across all specialist groups [20]. However, other studies have noted widespread dissatisfaction and challenges associated with remuneration for eConsult [22]. While it is vital to ensure that specialists are satisfied with a remuneration model, it is equally important to explore the preferences of the different models on patients, PCPs, and the payer [23]. For example, while patients may prefer a remuneration model where their PCP receives the quickest answer, this could result in poorer answer quality from specialists. Conversely, PCPs may prefer a model where they are remunerated, but this may result in increased utilization and higher costs to the payer. As eConsult services become more readily adopted, it will be necessary for policymakers and researchers to continually evaluate and monitor the impact of different remuneration models in order to ensure eConsult services remain sustainable.

Our study has several strengths. We used data from the Champlain BASE eConsult service, which is well established in our health region. The number of specialty groups available and the distribution of specialists are now at a steady state, allowing us to make meaningful inferences. The remuneration models we compared represent real world models that are either in use or have been suggested, which increases the applicability of our estimates.

Our study has some limitations. We simulated the impact of various remuneration models based on existing data collected using one specific remuneration model. Importantly, we do not observe how differently specialists/PCPs would behave under the different remuneration models. For example, it is possible that under the incentive model, specialists would respond to the bonus structure and expedite their work, which would increase the overall cost. Likewise, PCPs may submit more eConsults under the fee-for-service model, as they would receive compensation for each. Finally, we acknowledge that our paper only explores four payment models based on our experiences within our health system context, which is a single-payer system offering universal healthcare coverage.

Future research should address the impact of specialist remuneration models on health outcomes. Remuneration models may have an effect on specialist response times, quality of responses, avoidance of referrals, and patient and provider satisfaction. In addition, other payment models (e.g., capitated payment, models tied to quality-based care metrics) should be explored.

\section{Conclusions}

The prorated hourly rate model was found to be the most cost effective given the utilization and usage patterns of the Champlain BASE eConsult service. Paying more for an expedited response was not beneficial given the observed response times. Paying a flat fee each week was not cost effective due to the breadth of specialty groups available and heterogeneity in the number of eConsults submitted to each specialty. To our knowledge, this is the first study comparing remuneration models for eConsult services. While these results are based on a Canadian healthcare system and the current workflow of the Champlain BASE eConsult, our findings can inform policymakers considering the implementation of an eConsult service, or those wishing to further develop an existing platform In a different healthcare context where universal healthcare coverage is not offered, other remuneration models beyond the scope of the Champlain BASE eConsult data may be of interest. Future research should explore payment models for eConsult in other healthcare contexts and their impact on quality and outcome.

Acknowledgments: Financial support for this study came from the Ministry of Health and Long-Term Care INSPIRE-PHC Applied Health Research Question.

Author Contributions: C.L., E.K. and A.A. conceived and designed the study. C.D.A., P.D. and F.M. analyzed the data. All authors helped write and edit the paper.

Conflicts of Interest: The authors declare no conflict of interest. 


\title{
Abbreviations
}

\author{
PCP Primary care provider \\ BASE Building Access to Specialists through eConsultation
}

\section{References}

1. Barua, B.; Esmail, N. Waiting Your Turn: Wait Times for Health Care in Canada; Fraser Institute: Vancouver, BC, Canada, 2013.

2. Vidberg, N.; Forsberg, B.C.; Borowitz, M.; Molin, R. International comparisons of waiting times in health care-Limitations and prospects. Health Policy 2013, 112, 53-61. [CrossRef] [PubMed]

3. Kim-Hwang, J.E.; Chen, A.H.; Bell, D.S.; Guzman, D.; Yee, H.F., Jr.; Kushel, M.B. Evaluating electronic referrals for specialty care at a public hospital. J. Gen. Int. Med. 2010, 25, 1123-1128. [CrossRef] [PubMed]

4. North, F.; Uthke, L.D.; Tulledge-Scheitel, S.M. Integration of e-consultations into the outpatient care process at a tertiary medical centre. J. Telemed. Telecare 2014, 20, 221-229. [CrossRef] [PubMed]

5. Harno, K.; Paavola, T.; Carlson, C.; Viikinkoski, P. Patient referral by telemedicine: Effectiveness and cost analysis of an Intranet system. J. Telemed. Telecare 2000, 6, 320-329. [CrossRef] [PubMed]

6. Keely, E.; Liddy, C.; Afkham, A. Utilization, Benefits, and Impact of an e-Consultation Service across Diverse Specialties and Primary Care Providers. Telemed. J. e-Health 2013, 19, 733-738. [CrossRef] [PubMed]

7. Chen, A.H.; Murphy, E.J.; Yee, H.F., Jr. eReferral-A new model for integrated care. N. Engl. J. Med. 2013, 368, 2450-2453. [CrossRef] [PubMed]

8. Straus, S.G.; Chen, A.H.; Yee, H.F., Jr.; Kushel, M.B.; Bell, D.S. Implementation of an electronic referral system for outpatient specialty care. AMIA Ann. Symp. Proc. 2011, 2011, 1337-1346.

9. Eminovic, N.; de Keizer, N.F.; Wyatt, J.C.; ter Riet, G.; Peek, N.; van Weert, H.C.; Bruijnzeel-Koomen, C.A.; Bindels, P.J. Teledermatologic consultation and reduction in referrals to dermatologists: A cluster randomized controlled trial. Arch. Dermatol. 2009, 145, 558-564. [CrossRef] [PubMed]

10. Liddy, C.; Afkham, A.; Drosinis, P.; Joschko, J.; Keely, E. Impact of and satisfaction with a new eConsult service: A mixed methods study of primary care providers. J. Am. Board Fam. Med. 2015, 28, 394-403. [CrossRef] [PubMed]

11. Pak, H.S.; Datta, S.K.; Triplett, C.A.; Lindquist, J.H.; Grambow, S.C.; Whited, J.D. Cost minimization analysis of a store-and-forward teledermatology consult system. Telemed. J. e-Health 2009, 15, 160-165. [CrossRef] [PubMed]

12. Whited, J.D.; Hall, R.P.; Foy, M.E.; Marbrey, L.E.; Grambow, S.C.; Dudley, T.K.; Datta, S.; Simel, D.L.; Oddone, E.Z. Teledermatology's impact on time to intervention among referrals to a dermatology consult service. Telemed. J. e-Health 2002, 8, 313-321. [CrossRef] [PubMed]

13. Liddy, C.; Rowan, M.S.; Afkham, A.; Maranger, J.; Keely, E. Building access to specialist care through e-consultation. Open Med. 2013, 7, e1-e8. [PubMed]

14. Chen, A.H.; Kushel, M.B.; Grumback, K.; Yee, H.F., Jr. Practice profile. A safety-net system gains efficiencies through 'eReferrals' to specialists. Health Aff. 2010, 29, 969-971. [CrossRef] [PubMed]

15. Whited, J.D.; Datta, S.; Hall, R.P.; Foy, M.E.; Marbrey, L.E.; Grambow, S.C.; Dudley, T.K.; Simel, D.L.; Oddone, E.Z. An economic analysis of a store and forward teledermatology consult system. Telemed. J. e-Health 2003, 9, 351-360. [CrossRef] [PubMed]

16. Vimalananda, V.G.; Gupte, G.; Seraj, S.M.; Orlander, J.; Berlowitz, D.; Fincke, B.G.; Simon, S.R. Electronic consultations (e-consults) to improve access to specialty care: A systematic review and narrative synthesis. J. Telemed. Telecare 2015, 21, 323-330. [CrossRef] [PubMed]

17. Liddy, C.; Maranger, J.; Afkham, A.; Keely, E. Ten steps to establishing an e-consultation service to improve access to specialist care. Telemed. J. e-Health 2013, 19, 982-990. [CrossRef] [PubMed]

18. Bains, N.; Dall, K.; Hay, C.; Pacey, M.; Sarkella, J.; Ward, M. Population Health Profile: Champlain LHIN; Ministry of Health and Long-Term Care: Toronto, ON, Canada, 2008.

19. Ministry of Health and Long-Term Care. Schedule of Benefits for Physician Services under the Health Insurance Act; Ministry of Health and Long-Term Care: Toronto, ON, Canada, 2015.

20. Keely, E.; Drosinis, P.; Afkham, A.; Liddy, C. Perspectives of Champlain BASE Specialist Physicians: Their Motivation, Experiences and Recommendations for Providing eConsultations to Primary Care Providers. Stud. Health Technol. Inform. 2015, 209, 38-45. [PubMed] 
21. Berghout, R.M.; Eminovic, N.; de Keizer, N.F.; Birnie, E. Evaluation of general practitioner's time investment during a store-and-forward teledermatology consultation. Int. J. Med. Inform. 2007, 76, S384-S391. [CrossRef] [PubMed]

22. Armstrong, A.W.; Kwong, M.W.; Ledo, L.; Nesbitt, T.S.; Shewry, S.L. Practice models and challenges in teledermatology: A study of collective experiences from teledermatologists. PLoS ONE 2011, 6, e28687. [CrossRef] [PubMed]

23. Devlin, R.A.; Sarma, S.; Hogg, W. Remunerating primary care physicians: Emerging directions and policy options for Canada. Healthc. Q. 2005, 9, 34-42. [CrossRef]

(C) 2016 by the authors; licensee MDPI, Basel, Switzerland. This article is an open access article distributed under the terms and conditions of the Creative Commons Attribution (CC-BY) license (http:/ / creativecommons.org/licenses/by/4.0/). 